\title{
NEAR-INFRARED SPECTRA OF ARP 220: SPATIALLY RESOLVED CO ABSORPTION IN THE INNER KILOPARSEC
}

\author{
L. ARMus, G. Neugebauer, B. T. SoIfER, AND K. Matthews \\ Palomar Observatory, California Institute of Technology, 320-47, Pasadena, California 91125 \\ Received 1995 April 17; revised 1995 August 9
}

\begin{abstract}
Near-infrared spectra covering $\lambda \sim 2.0-2.5 \mu \mathrm{m}$ with a spectral resolution of $R \sim 80$ and a spatial resolution of $0.7^{\prime \prime}$ have been obtained from two long slits oriented at a position angle of $96^{\circ}$, centered on and $2.0^{\prime \prime}$ south of the two near-infrared nuclei in the ultraluminous infrared galaxy Arp 220. These data have allowed us to determine the strengths of the stellar $\mathrm{CO}$ absorption feature and the $B \gamma$ emission line over the central 7 $\operatorname{arcsec}(2.5 \mathrm{kpc})$ on scales of $\sim 250 \mathrm{pc}$. The two near-infrared nuclei have very strong stellar atmospheric CO absorption coupled with very red $H-K$ colors, implying significant contributions $(40 \%-50 \%)$ to the $K$-band continua from young, red supergiant stars formed in a recent burst of star formation. In contrast to the strong nuclear $\mathrm{CO}$ absorption, the equivalent width of the $B \gamma$ emission line is weak, and peaked at the position of the eastern infrared nucleus where the equivalent width is $\sim 1.0 \mathrm{~nm}$. There is no detectable $B \gamma$ emission in the spectra extracted from the southern slit. We suggest that the youngest part of the starburst $\left(t \sim 7-8 \mathrm{Myr}\right.$ old) is confined to the Arp 220 nuclei, while the surrounding region (out to at least $2.0^{\prime \prime}-2.5^{\prime \prime}$ radius) is dominated by older stars $\left(\sim 10^{8} \mathrm{yr}\right)$ associated with a previous burst. Together these components are able to account for less than $10 \%$ of the bolometric luminosity of Arp 220. The dominant energy source in Arp 220 apparently remains hidden even at $2.2 \mu \mathrm{m}$. () 1995 American Astronomical Society.
\end{abstract}

\section{INTRODUCTION}

Arp 220 (UGC 9913) is the closest (72 Mpc for $H_{0}=75$ $\mathrm{km} \mathrm{s}^{-1} \mathrm{Mpc}^{-1}$ ) and most intensely studied member of the class of ultraluminous infrared galaxies (ULIRGs) defined by Sanders et al. (1988) and Soifer et al. (1989) to have $L_{\mathrm{IR}} \geqslant 10^{12} L_{\odot}$. It has been suggested by Sanders et al. that the ULIRGs represent an intermediate stage in the evolution of a pair of merging, spiral galaxies into an optically visible quasar. Arp 220 fits nicely into this model with its highly irregular optical morphology (Arp 1966), double nucleus (Graham et al. 1990, hereafter referred to as G90) and LINER-like optical spectrum. It is often referred to as the prototype of the ULIRG class.

A powerful starburst provides a natural link between the merger process and the generation of a central AGN, as the inflowing gas dissipates energy and angular momentum, forming molecular clouds, and finally massive stars on the kiloparsec scale. The gas not involved in the starburst may directly fuel a nascent central black hole, or the stellar remnants may coalesce into a massive blackhole themselves at the end of the starburst phase. The strong galactic winds thought to be an integral part of the starburst may aid in uncovering the young active nucleus as they blow left over material from the area surrounding the central engine into the galactic halo and possibly into the intergalactic medium.

Although there is evidence that an optically buried AGN may exist in Arp 220 (the most recent being the VLBI work of Lonsdale et al. 1993, 1995), there is similarly strong evidence that enhanced star formation is ongoing. The fraction of the bolometric luminosity contributed by the starburst, however, remains uncertain. There is a very large reservoir of molecular gas, $\sim 2 \times 10^{10} \mathscr{H}_{\odot}$, in the central $10^{\prime \prime}$ (Scoville et al. 1991) which would provide adequate fuel for an in- tense starburst. There is also strong PAH emission at $3.3 \mu \mathrm{m}$ (Rieke et al. 1985), generally associated with starbursts and not AGN, as well as strong $2.3 \mu \mathrm{m} \mathrm{CO}$ absorption from the atmospheres of cool, young, supergiant stars (Rieke et al.; Ridgway et al. 1994). Starburst models with exponentially declining star formation rates and ages of 30-100 Myr have been fit to a wide variety of observations by Rieke et al. (1985). These models imply a starburst may contribute up to $50 \%$ of the bolometric luminosity. Mazzarella et al. (1992) show that the near-infrared colors in Arp 220 at radii of $1^{\prime \prime}-2^{\prime \prime}$ are similar to those seen in dusty starburst galaxies like M82 and NGC 253. Extended $15 \mathrm{GHz}$ emission on scales of one-few arcseconds is given by Sopp \& Alexander (1991) as proof that a starburst is active in the immediate circumnuclear environment of Arp 220. Hubble Space Telescope $V, R$, and $I$-band images (Shaya et al. 1994) contain a number of clumps within the inner kiloparsec which may be luminous stellar associations with luminosities greater than $10^{9} L_{\odot}$. In addition to this evidence for young stars, optical spectra show strong $\mathrm{H} \beta$ absorption lines indicating the presence of a large, intermediate age $\left(\sim 10^{8} \mathrm{yr}\right)$ population of stars in and around the nuclei (Rieke et al. 1985; Armus et al. 1989). There is thus a wealth of suggestive evidence for a starburst occurring in Arp 220. However, since most of these observations have been made with large $\left(5-10^{\prime \prime}\right)$ beams, there is little direct spatial information on the distribution of young stars and the size of the region where the starburst is occurring.

In order to measure the strength of the $\mathrm{CO}$ absorption as well as the $B \gamma$ and $\mathrm{H}_{2}$ emission features throughout the central kiloparsec of Arp 220 we have obtained long-slit spectra centered on, and offset from, the twin infrared nuclei imaged by G90. These data allow us to construct two, onedimensional maps of the nuclear starburst on scales of 300- 


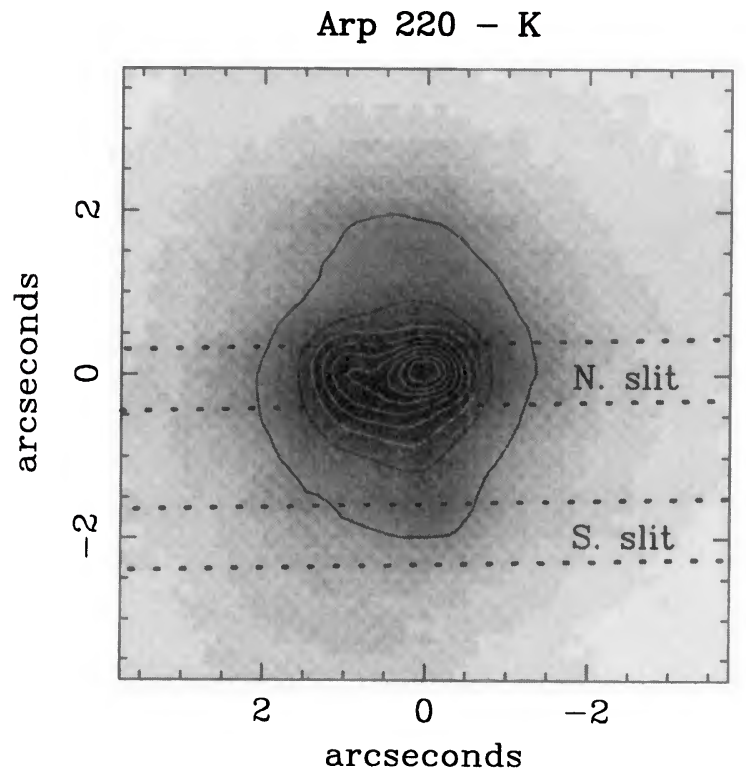

FIG. 1. $K$-band image of the central $7.5^{\prime \prime} \times 7.5^{\prime \prime}$ of Arp 220 showing the positions of the northern and southern slits. North is up and east is to the left. The western infrared nucleus has been placed at 0,0 . Contours are linear, progressing from $10 \%$ to $90 \%$ of the peak pixel in steps of $10 \%$.

$400 \mathrm{pc}$. In Sec. 2 we describe the observations and the data reduction. In Sec. 3 we discuss the results in detail, and in Sec. 4 we discuss the source of the strong $\mathrm{CO}$ absorption in the Arp 220 nucleus and its implication for the total power output of this galaxy.

\section{OBSERVATIONS AND DATA REDUCTION}

The observations reported here were obtained on the night of 1994 May 2 (UT) using the grism mode of the nearinfrared camera (NIRC) mounted at the $f / 25$ forward Cassegrain focus of the W. M. Keck telescope. The NIRC employs a Hughes-SBRC InSb photovoltaic array made up of $256 \times 256,30 \mu \mathrm{m}$ square pixels. At the $f / 25$ focus, the pixels correspond to $0.15^{\prime \prime} \times 0.15^{\prime \prime}$ on the sky. See Matthews \& Soifer (1994) for a detailed description of the camera.

Grism spectra covering the 1.4-2.5 $\mu \mathrm{m}$ wavelength region were obtained with a $38^{\prime \prime} \times 0.7^{\prime \prime}$ slit at two positions on the galaxy. The first intercepted light from the two nearinfrared nuclei, while the second was offset $2.0^{\prime \prime}$ to the south. The position angle of the slit in both cases was $96^{\circ}$. Figure 1 shows a schematic representation of the slits placed on a $K$-band image of Arp 220 taken immediately before the spectra were obtained. The slit locations were determined by imaging Arp 220 with the slits and the broadband $K$ filter in place. With the $0.7^{\prime \prime}$ wide slit, the spectral resolution $(\lambda / \Delta \lambda)$ is $\sim 80$, with a dispersion of $0.603 \mathrm{~nm} /$ pixel. Five integrations of $200 \mathrm{~s}$ each were taken on the nuclei. The galaxy was moved $6.0^{\prime \prime}$ along the slit between successive integrations to allow accurate sky subtraction. In addition, three spectra, each of $240 \mathrm{~s}$ duration, were taken through the slit positioned $2.0^{\prime \prime}$ to the south of the nuclei. For these latter observations, the sky position was taken as a blank area of the sky $50^{\prime \prime}$ to the south of the Arp 220 nuclei. The star HD5659 (G5 V), observed immediately following Arp 220, was used to re- move the instrumental response and the telluric absorption features from the galaxy spectra. Individual spectra were wavelength calibrated based upon strong night sky and astronomical (the planetary nebula NGC 7027) emission lines. Seeing at the time of these observations was approximately $0.7^{\prime \prime}$ (full width at half maximum-FWHM), as determined from stellar images taken immediately before and after the Arp 220 spectra. The $K$-band spectra covering the 2.0-2.5 $\mu \mathrm{m}$ region are presented in this paper. Although we derive $H-K$ colors from these spectra, the detailed description of the $H$-band spectra will be presented in a subsequent paper, as will the [Fe II] $1.644 \mu \mathrm{m}$ and $\mathrm{H}_{2}$ emission features. Here, we focus on the strengths and spatial distributions of the $\mathrm{CO}$ absorption band and the $B \gamma$ emission feature.

\section{RESULTS}

From the two-dimensional long-slit data, we have extracted a set of one-dimensional spectra over the central 7.5" for detailed analysis of the variation of spectral slope, and emission and absorption line strength as a function of position. From the northern slit data, spectra have been extracted in $0.45^{\prime \prime}$ wide bins, each separated by $0.45^{\prime \prime}$. From the southern slit data, spectra have been extracted in $0.75^{\prime \prime}$ wide bins, each spaced by $0.75^{\prime \prime}$. In Fig. 2, eight representative spectra (four each from the northern and southern slit data) are displayed. Arp 220 shows spatially variable $\mathrm{CO}$ absorption and $B \gamma$ emission over the central seven arcseconds. The 1-0 S(1) line of $\mathrm{H}_{2}$ at $2.121 \mu \mathrm{m}$ is also prominent in these spectra, and the 1-0 S(0) line can be seen as a weak feature at $2.223 \mu \mathrm{m}$. The broad feature at $\sim 2.42 \mu \mathrm{m}$ may have a large contribution from the 1-0 $Q$ branch lines of $\mathrm{H}_{2}$ at 2.4-2.45 $\mu \mathrm{m}$, but the proximity of these lines to the edge of the $K$-band window prohibits a quantitative measurement. As mentioned in Sec. 1, the strengths of the $\mathrm{H}_{2}$ features, along with the $H$-band spectral data will be discussed fully in a subsequent paper.

The spectroscopic $\mathrm{CO}$ index, $\mathrm{CO}_{\mathrm{sp}}$, is usually defined as $\mathrm{CO}_{\text {sp }}=-2.5 \log R$, where $R$ is the average of the ratio of the flux density over the $2.31-2.40 \mu \mathrm{m}$ range to the extrapolated continuum (e.g., Doyon et al. 1994; Ridgway et al. 1994). To obtain accurate fits to the Arp 220 spectra over the entire 2.0-2.4 $\mu \mathrm{m}$ wavelength range, we fit each extracted spectrum to the functional form:

$$
f_{\lambda}=k \lambda^{\beta} e^{A \tau(\lambda)}
$$

over the rest wavelength range $2.065-2.380 \mu \mathrm{m}$, where $f_{\lambda}$ is flux density, $\lambda$ is wavelength, and $k, \beta$, and $A$ are constants. The parameter $\tau(\lambda)$ is defined to have the shape of the CO absorption in the western infrared nucleus over the wavelength range of $2.256-2.380 \mu \mathrm{m}$, and to be zero from 2.065 to $2.256 \mu \mathrm{m}$. It is normalized to unity at $2.35 \mu \mathrm{m}$. In every other spectrum the shape of the CO absorption is fixed, but $k$, $\beta$, and $A$ are allowed to vary. In each case we have excluded the $\mathrm{H}_{2}$ and $B \gamma$ emission features from the continuum fit to allow an accurate determination of the spectral slope, $\beta$. This method implicitly assumes that only the strength of the $\mathrm{CO}$ absorption (and not the shape of the band) changes as a function of position along the slit. From these spectral fits we 

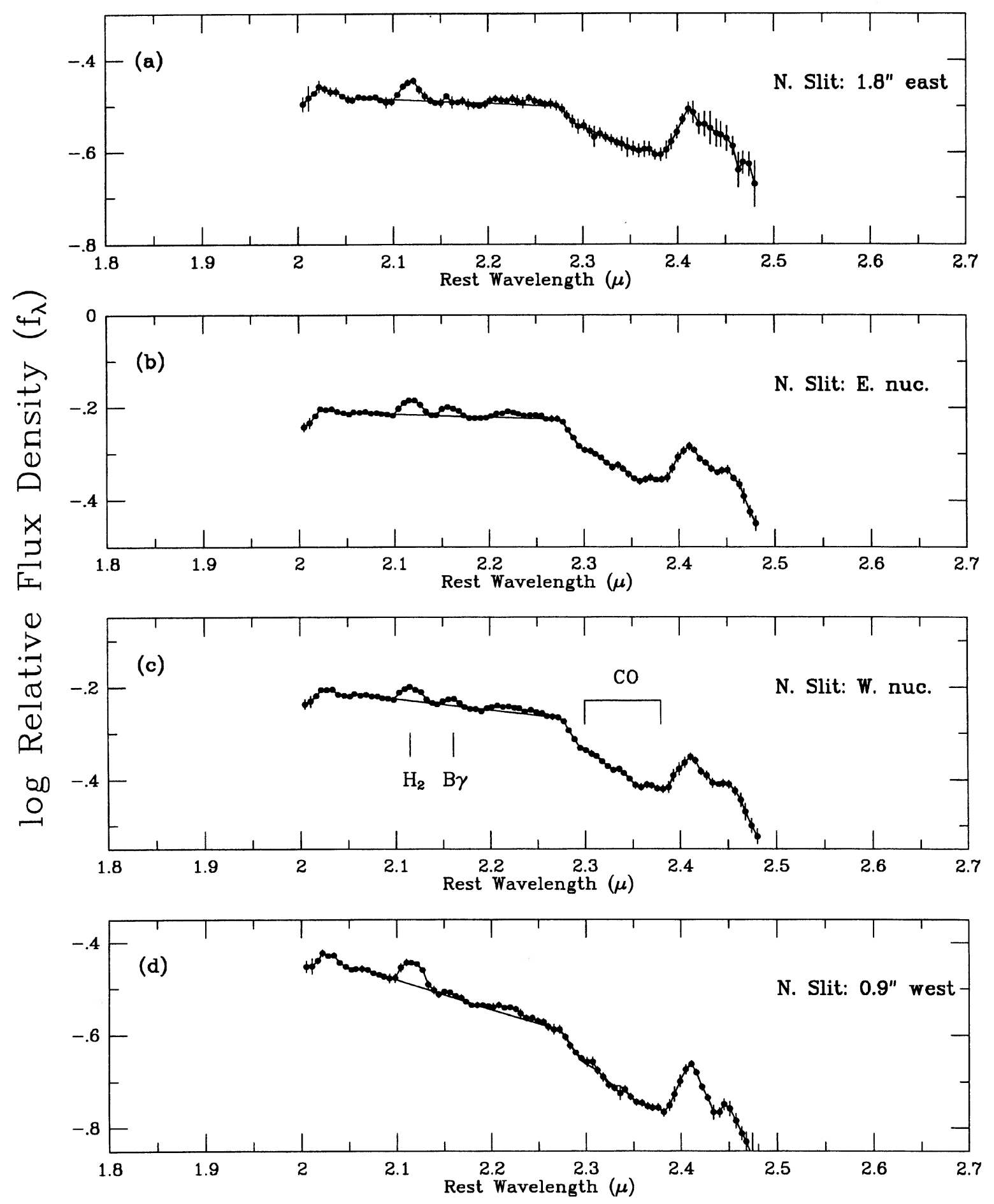

FIG. 2. Eight spectra extracted from the long-slit data. Four of these, (a)-(d), are taken from the northern slit which runs through the two near-infrared nuclei. The remaining four spectra, (e)-(h), are taken from the southern slit which runs parallel to the northern slit but displaced $2.0^{\prime \prime}$ to the south. All spectra are plotted on a linear (rest wavelength), log (relative flux density per unit wavelength interval) scale. The northern slit spectra are each $0.45^{\prime \prime}$ wide, while the southern slit spectra are each $0.75^{\prime \prime}$ wide. The centroids of the spectra are shown in the upper right of each plot, with displacements in right ascension measured from the position of the western infrared nucleus. The positions of the $\mathrm{H}_{2}[1-0 S(1)]$ and $B \gamma$ emission lines and the CO absorption band are marked in (c), the spectrum extracted from the position coincident with the western infrared nucleus. Although not marked, the $1-0 Q$ branch lines of $\mathrm{H}_{2}$ are visible in the $2.4-2.45 \mu \mathrm{m}$ region. The proximity of these lines to the edge of the band makes any quantitative measurements difficult. 

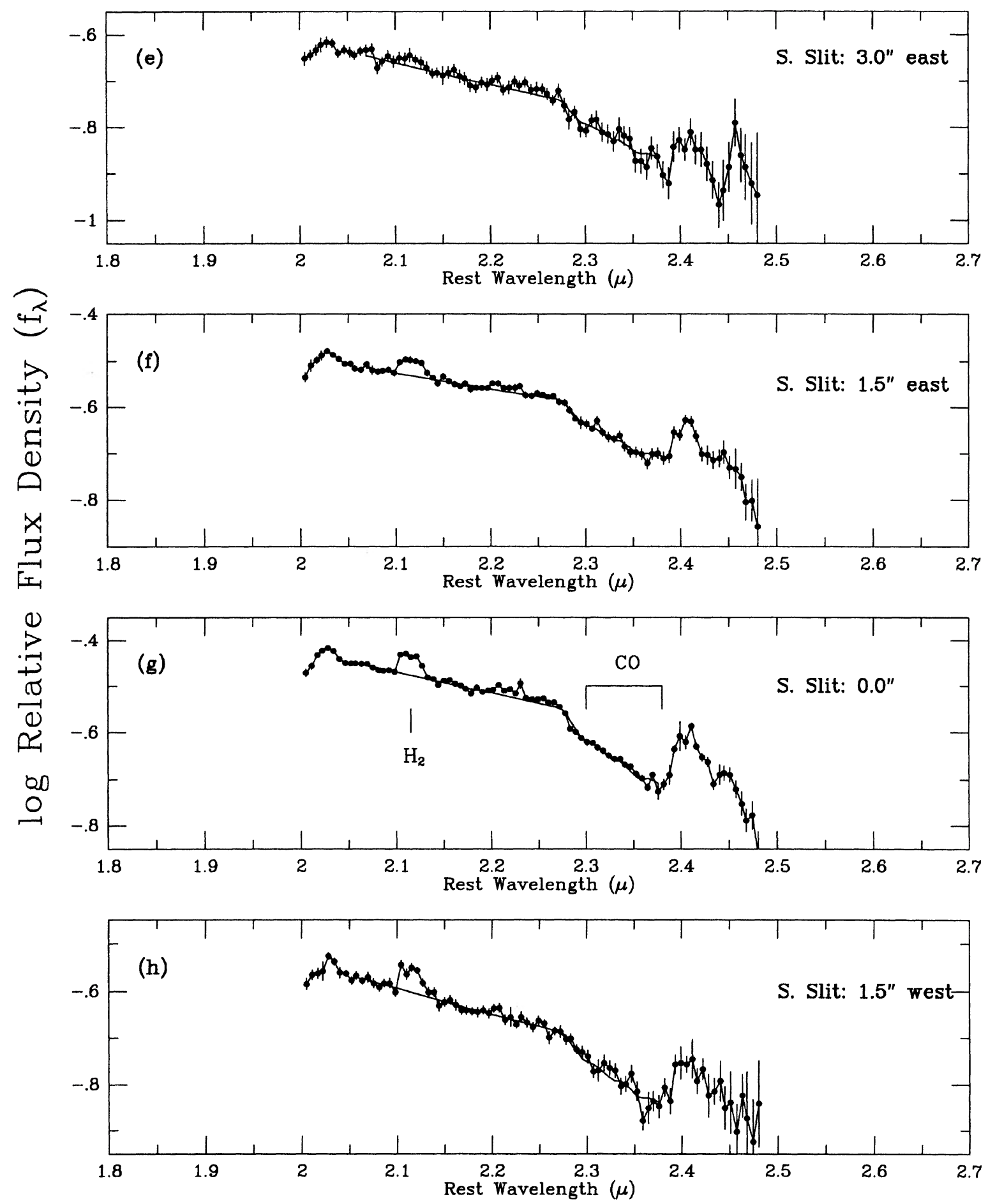

FIG. 2. (continued)

have derived the spectroscopic $\mathrm{CO}$ index, $\mathrm{CO}_{\mathrm{sp}}$, which is found to be $A / 1.11$, with a variation of less than $1 \%$ over the full range of values. In these fits we have neglected the contribution from the 4-3 $S(3), 2-1 S(0)$, and 3-2 $S(1)$ emission lines of $\mathrm{H}_{2}$ which, at $2.344,2.355$, and $2.386 \mu \mathrm{m}$, respectively, fall within the $\mathrm{CO}$ absorption band. There is no evidence for these emission lines in the spectra. For collisionally excited $\mathrm{H}_{2}$ emission, these lines are less than $1 \%-2 \%$ of the 1-0 S(1) line (Black \& van Dishoeck 1987). In regions where fluorescent excitation dominates, the 2-1 $S(0)$ and 3-2 $S(1)$ lines can reach values of about $25 \%$ of the 1-0 $S(1)$ line. However, under these conditions the 2-1 $S(1)$ 
line $(2.247 \mu \mathrm{m})$ is expected to be greater than $50 \%$ of the strength of the 1-0 S(1) line (Black \& van Dishoeck). This is clearly not the case in the Arp 220 spectra (see Fig. 2). It is likely, therefore, that the $\mathrm{H}_{2}$ emission is collisionally excited and the contributions of the 4-3 $S(3), 2-1 S(0)$, and 3-2 $S(1)$ lines to the $\mathrm{CO}$ absorption band are negligible.

In Figs. 3(a)-3(d), we show the relative continuum flux density at $2.1 \mu \mathrm{m}$, the spectroscopic $\mathrm{CO}$ index $\mathrm{CO}_{\mathrm{sp}}$, the power-law spectral index $(\beta)$, and the $B \gamma$ emission line equivalent width (EQW), as a function of position along the slit(s). In all panels of Fig. 3 the zero point in R.A. is taken as the position of the western infrared nucleus. Although the spectroscopic slit used was $38^{\prime \prime}$ in length, we have plotted only the region over which we have a reliable measurement of the $\mathrm{CO}$ index. In Fig. 3(b) we also indicate the range of $\mathrm{CO}_{\mathrm{sp}}$ values for $50 \mathrm{E}$ and $\mathrm{S} 0$ galaxies taken from the work of Frogel et al. (1978), converted from a photometric to a spectroscopic $\mathrm{CO}$ index using $\mathrm{CO}_{\mathrm{sp}}=1.46 \mathrm{CO}_{\mathrm{ph}}-0.02$ (Doyon et al. 1994), where $\mathrm{CO}_{\mathrm{ph}}$ is the photometric $\mathrm{CO}$ index. The average $\mathrm{CO}_{\mathrm{sp}}$ of these nuclei (dominated by giant stars) is $0.18 \pm 0.04$ mag.

The CO index shows a broad peak centered roughly between the two infrared nuclei in the northern slit. There are four positions along this slit which have $\mathrm{CO}$ indices clearly larger than those seen in the nuclei of galaxies dominated by old stars. Two of these are located at the positions of the infrared nuclei, one is located about $0.5^{\prime \prime}$ west of the western infrared nucleus, and the final position is located directly between the infrared nuclei. Since the seeing at the time of these observations was about $0.7^{\prime \prime}$ FWHM, the measured strength of the $\mathrm{CO}$ absorption midway between the infrared nuclei may be strongly affected by the nuclei themselves. Within the measured uncertainties $(0.01 \mathrm{mag})$ the eastern and western nuclei have similar $\mathrm{CO}$ indices of $\sim 0.27$ mag. Although there are no points along the southern slit with values of $\mathrm{CO}_{\text {sp }}$ larger than the range seen in E/SO nuclei, there is a rise in the strength of the index at the positions directly south of the infrared nuclei. At the point $2.0^{\prime \prime}$ south of the western infrared nucleus, the $\mathrm{CO}$ index reaches a peak of $\sim 0.22 \mathrm{mag}$.

The power-law continuum index $\beta$ shows a spatial behavior which is different than that seen in $\mathrm{CO}_{\mathrm{sp}}$. In Fig. 3(c) the northern slit spectra show a sharp rise in $\beta$ (flatter spectra) near the position of the eastern infrared nucleus. The eastern nucleus has a measurably flatter (redder) spectral slope $(\beta=-0.38 \pm 0.05)$ than the western nucleus, where $\beta=$ $-1.33 \pm 0.03$ [compare Figs. 2(b) and 2(c)]. Over the southern slit the change in the $\beta$ value is much more gradual. Here, the spectral slope rises to values of about -1.6 , south of the eastern nucleus, never becoming as red as the reddest spectra extracted from the northern slit.

The equivalent width of $B \gamma$ as a function of position along the northern slit is shown in Fig. 3(d). The $B \gamma$ emission line was not confidently detected anywhere along the southern slit. For all the extracted spectra the $B \gamma$ equivalent width was calculated from direct integration of the line flux between 2.147 and $2.170 \mu \mathrm{m}$ in the rest frame, taking a linear continuum fit to the data on either side of the line. Although the $B \gamma$ emission from Arp 220 is weak, there is a shallow, asymmetric peak in the equivalent width at the po- sition of the eastern infrared nucleus, where EQW $=1.0 \pm 0.1$ $\mathrm{nm}$. At the position of the western infrared nucleus the $B \gamma$ $\mathrm{EQW}=0.7 \pm 0.1 \mathrm{~nm}$. The $B \gamma$ line is not detected at radii larger than about $1^{\prime \prime}$. The nuclear $B \gamma$ line fluxes, calculated from our equivalent widths and the $K$-band magnitudes $(13.7$ and $13.2 \mathrm{mag}$ ) reported in $\mathrm{G} 90$, are 1.3 and $1.5 \times 10^{-15}$ ergs $\mathrm{cm}^{-2} \mathrm{~s}^{-1}$ for the eastern and western nucleus, respectively. These flux values are uncertain by approximately $25 \%$.

\section{DISCUSSION}

In this section we estimate, the importance of young stars to both the near-infrared and bolometric luminosity of Arp 220 , by using the strengths of the $\mathrm{CO}$ and $B \gamma$ features as well as the $H-K$ color. We begin with a general discussion on the power, and potential problems, of using these particular nearinfrared diagnostics as starburst indicators. We then attempt to remove the nonstellar fraction of the near-infrared light to isolate the fraction of stellar emission due to young stars. Finally, we compare the stellar continuum emission to that expected from aging starburst populations as calculated by Leitherer \& Heckman (1995). The models are used to assess the contribution of the starburst(s) to the bolometric light of Arp 220.

Red supergiants, which have $\mathrm{CO}_{\mathrm{sp}} \sim 0.3-0.5 \mathrm{mag}$ for types later than $\mathrm{M} 0$, can dominate the near-infrared spectrum of a starburst at an age of about $10^{7} \mathrm{yr}$. The $\mathrm{CO}$ absorption increases in strength with decreasing stellar temperature, increasing luminosity, and increasing metallicity. Doyon et al. (1994) show that $\Delta \mathrm{CO}=0.11 \Delta[\mathrm{Fe} / \mathrm{H}]$, where $[\mathrm{Fe} / \mathrm{H}]$ is the logarithm of the metal abundance relative to the Sun. This conversion implies that the strong $\mathrm{CO}$ absorption seen in the Arp 220 nuclei can be accounted for by giant stars if the metallicity were about five times greater than Solar. However, there are two reasons why this is not likely to be the case in Arp 220. First, as emphasized by Doyon et al., the $\mathrm{CO}$ index may be less sensitive to metallicity in high metallicity environments than implied by this relation because it is derived from data on low metallicity systems. The $\mathrm{CO}$ atmospheric band is made up of many saturated lines (McGregor 1987) and therefore it's strength may not respond to increases in the metallicity in a linear manner (i.e., as suggested by the above relation). Second, although strong starbursts are expected to enrich the ISM with heavy elements through stellar winds and supernovae, there is no compelling evidence for such a high metallicity in the nucleus of Arp 220. Furthermore, models suggest that increasing the metal abundance by such a large factor late in the life of a metalrich galaxy is highly unlikely (e.g., Matteucci \& Padovani 1993). We therefore suggest that the large CO band strength in Arp 220 reflects a change in the stellar population dominated by late-type supergiants.

Even if an unusually large $\mathrm{CO}$ index can be attributed to stars, care must be taken in interpreting the population mix. Late type dwarfs (later than M5) can have spectroscopic CO indices of up to $\sim 0.02 \mathrm{mag}$, but they also often have quite large absorption shortward of $2.2 \mu \mathrm{m}$ due to atmospheric $\mathrm{H}_{2} \mathrm{O}$ vapor. This type of absorption is not evident in the 

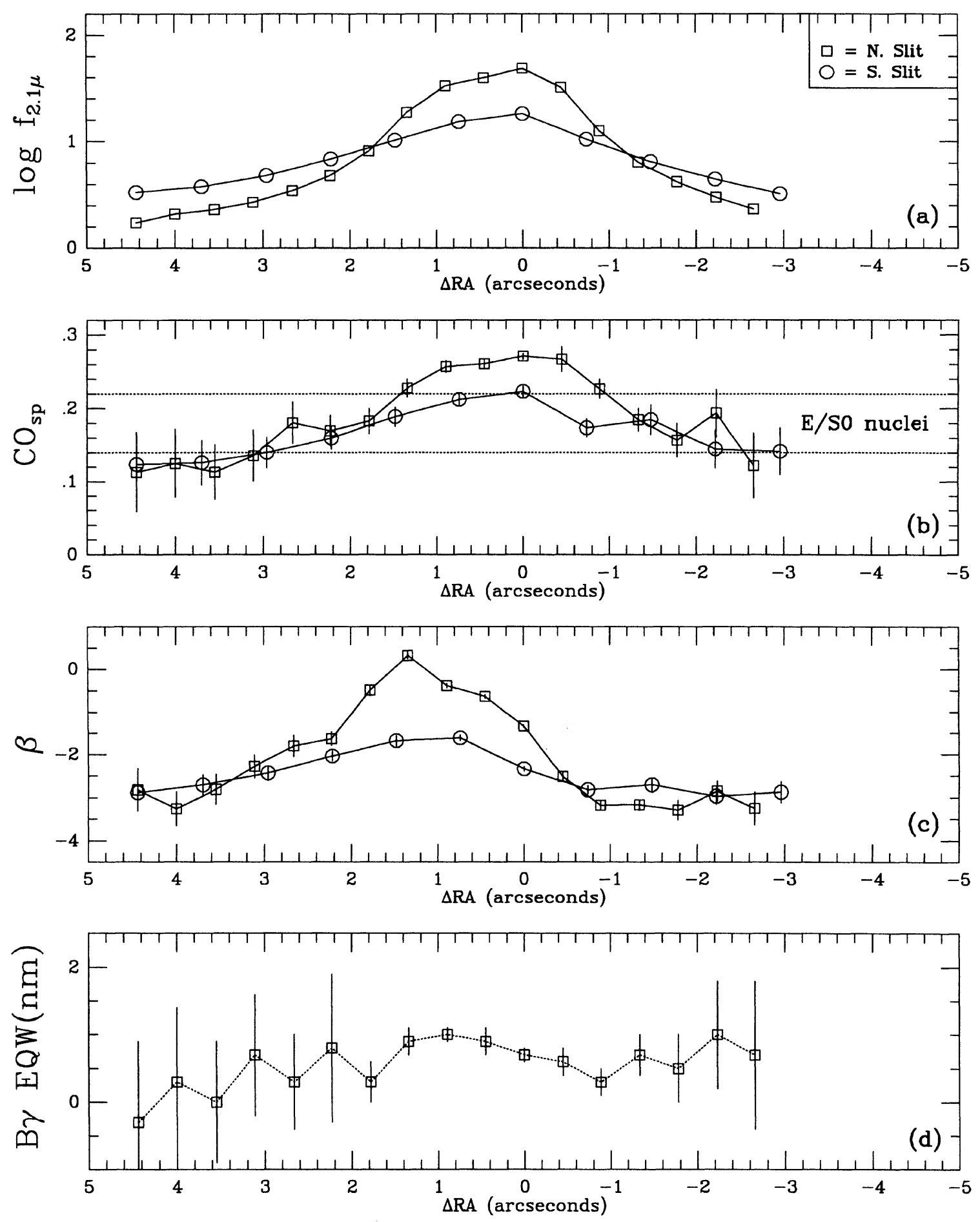

FIG. 3. The strength of various measured quantities vs position along both the northern and southern spectroscopic slits. In (a)-(c) the northern slit data points are designated with open squares and the southern slit data points are designated with open circles. The zero point in right ascension is located at the position of the western infrared nucleus. (a) is the log of the relative continuum flux density measured at a rest wavelength of $\sim 2.1 \mu \mathrm{m}$, where the northern and southern slit data have been normalized separately. For reference, before normalization the peak in the northern slit curve is a factor of about 9.4 greater than the peak in the southern slit curve. (b) is the spectroscopic CO index discussed in Sec. 3 (in magnitudes), with the locus of E/S0 nuclei taken from Frogel $e t$ al. (1978) shown as dotted lines. (c) is the power-law spectral index determined by fitting the line-free conintuum shortward of the CO absorption to the functional form $f_{\lambda} \propto \lambda^{\beta}$. The $B \gamma$ emission line equivalent width (in $\mathrm{nm}$ ) as a function of position along the northern slit is shown in (d). There are no detections of $B \gamma$ in the spectra extracted from the southern slit. 

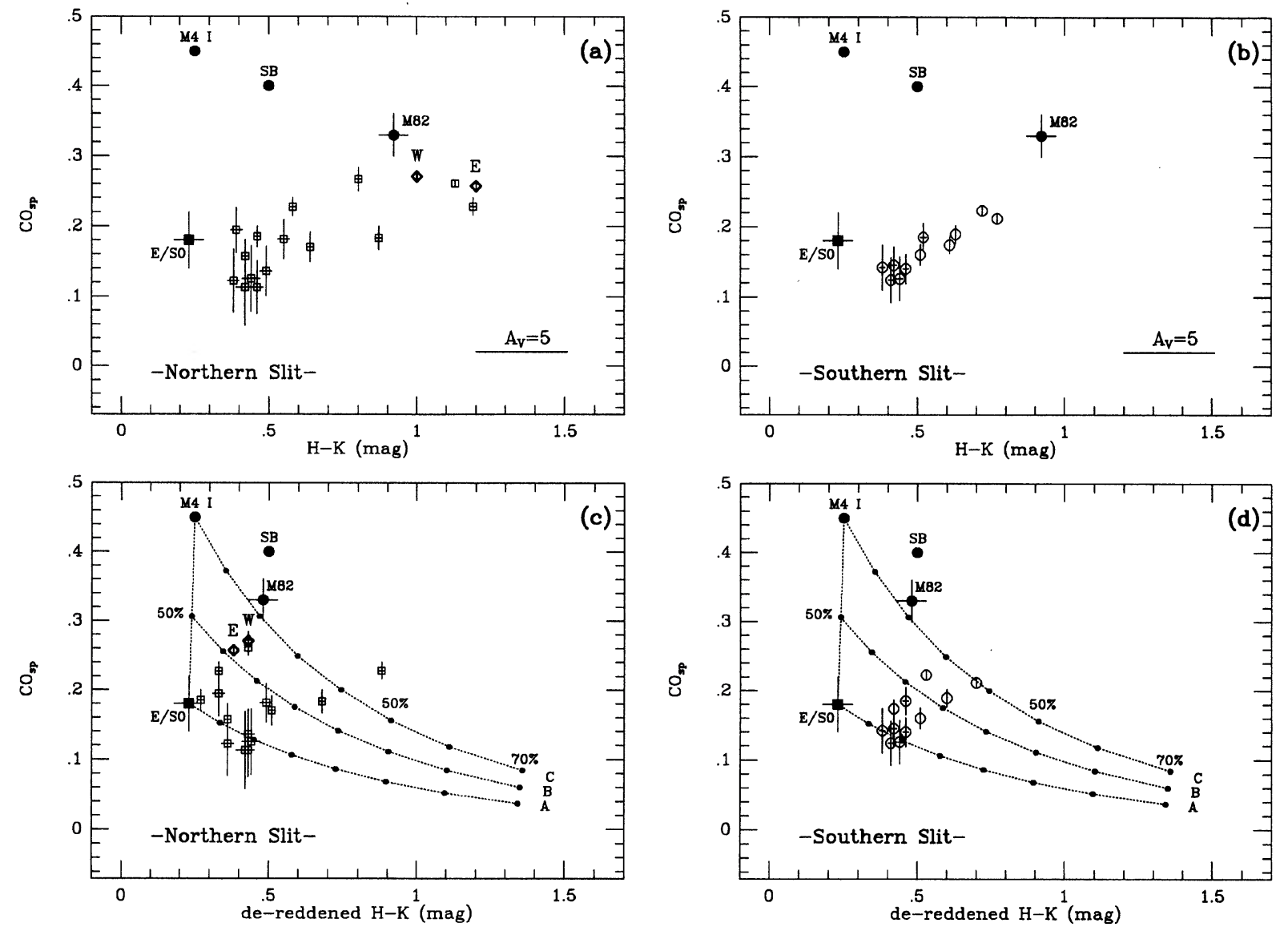

FIG. 4. The spectroscopic CO index vs the $H-K$ color for all extracted spectra plotted in Fig. 3. (a) shows the northern slit points (open squares) and (b) shows the southern slit points (open circles). In (a) and (c) the nuclei have been plotted as open diamonds and labelled "W" and "E" to denote the western and eastern infrared nuclei, respectively. The mean $\mathrm{CO}_{\mathrm{sp}}$ and $H-K$ value of a typical E/S0 galaxy (Frogel et al. 1978; Aaronson 1977) is plotted as a solid square. Filled circles mark the locations of the central two arcseconds of the starburst galaxy M82 (Larkin et al. 1994; Ridgway et al. 1994), and an M4 supergiant star (Frogel et al. 1978). The starburst model, labeled as SB, is a fading burst an age of 7 Myr with a Salpeter IMF over a mass range of 1-100 $\mathscr{H}_{\odot}$ (Leitherer \& Heckman 1995). The CO indices for each stellar type used in the synthesis have been taken from the compilation of Doyon et al. (1994). The SB, E/S0, and M4 I points include no extinction. A horizontal line in a \& b indicates the change in $H-K$ for an $A_{V}=5$ mag. In (c) $\&$ (d) the $H-K$ colors have been de-reddened following the prescription described in the text. The E/S0, M4 I, and SB points are dust free and thus have not been moved from their positions in (a) and (b). The vertical dotted line in Figs. (c) \& (d) represent the mixing curve of an old stellar population (the E/S0 point) and a pure red supergiant (the M4 I point). An equal mix of old stars and red supergiants is indicated by the 50\% point. The dotted curves labeled "A," "B," and "C" represent the effect of adding warm $(600 \mathrm{~K})$ dust emission to this stellar population mix. Curve " $\mathrm{A}$ " adds warm dust to a pure old stellar population. Curve "B" adds warm dust to a population with a $\mathrm{CO}_{\mathrm{sp}}$ and $H-K$ derived by mixing old stars and a pure M4 I star in equal amounts. Curve " $\mathrm{C}$ " adds warm dust to pure M4 supergiant light. The three model curves are marked off in units of $10 \%$ of the total $K$-band light, with selected fractions (50\% and $70 \%)$ labeled. In all cases the dust emissivity is taken to be proportional to $\lambda^{-2}$.

Arp 220 data, and thus late dwarfs are probably not responsible for the strong $\mathrm{CO}$ absorption. Late giants (e.g., later than $\mathrm{M} 4$ or M5) can have $\mathrm{CO}_{\mathrm{sp}} \geqslant 0.30 \mathrm{mag}$, comparable to the large $\mathrm{CO}_{\mathrm{sp}}$ seen in the nuclei of luminous starburst galaxies (e.g., Ridgway et al. 1994). Values of $\mathrm{CO}_{\mathrm{sp}}$ in the range 0.3-0.35 mag, therefore, cannot be taken as a priori evidence for a population of red supergiant stars. On the other hand, relatively low $\mathrm{CO}$ indices do not rule out a starburst, since emission from dust dilutes the measured $\mathrm{CO}$ absorption in an integrated spectrum. Extinction, while not affecting the strength of the $\mathrm{CO}$ absorption directly, does affect the estimated dust contribution, and thus can indirectly affect the derived intrinsic strength of the $\mathrm{CO}$ index. With these caveats in mind, the strengths of the $\mathrm{CO}$ band and the $B \gamma$ emission lines, together with the near-infrared colors, can help constrain the parameters of a burst.

In Figs. 4(a) and 4(b) we plot $\mathrm{CO}_{\text {sp }}$ vs $H-K$ for the northern and southern slit spectra. For comparison, we include the positions of a typical E/SO galaxy (Frogel et al. 1978; Aaronson 1977), the central 2.0" (30 pc) of the starburst galaxy M82 (Larkin et al. 1994; Ridgway et al. 1994), and a starburst model (Leitherer \& Heckman 1995) having an initial mass function (IMF) slope of $\alpha=2.35$ (Salpeter 1955), a stellar mass range of $1-100 \mathscr{C}_{\odot}$, and an age of 7 Myr. All the Arp 220 points have a $\mathrm{CO}_{\text {sp }}$ smaller than that found by Ridgway et al. for the prototype starburst galaxy M82.

There is clearly a large spread in the $H-K$ color among 
the Arp 220 points, as first pointed out by Mazzarella et al. (1992). As noted above, extinction and dust emission, both expected to be associated with a nuclear starburst, will redden the $H-K$ colors and weaken the measured $\mathrm{CO}$ index. To estimate the fraction of the $K$-band light contributed by young stars, the effects of dust emission and extinction must therefore be removed.

For the nuclear points, we use the extinction as determined from observations of the near-infrared $B \gamma$ and $P \beta$ emission lines (Larkin et al. 1995), which measure an $A_{V}=9.8$ and 12.9 mag for the western and eastern nucleus, respectively.

For the off-nuclear points, where emission-line flux ratio data are not available, we use the color maps of Mazzarella et al. (1992) to estimate the $A_{V}$ values at the positions of our extracted spectra. Although there is evidence that the seeing at the time of the former observations was slightly better than for the data here, we are using the color maps to estimate the off-nuclear extinctions only, where the seeing is far less critical. For each extracted spectra, we measure the $J-H$ and $H-K$ color at the corresponding location in the Mazzarella et al. map and use this to derive an extinction. Each off-nuclear point was assumed to have $J-H$ and $H-K$ colors dominated by three processes-atmospheric emission from old stars, extinction from interstellar dust in a foreground screen, and emission from warm dust at $600 \mathrm{~K}$. The estimated extinction ranges from $A_{V}=0$ to $4 \mathrm{mag}$ for the off-nuclear points. Note that including an obscuring screen of dust is only done to remove the reddening effect of the dust from the observed $H-K$ color. We are not explicitly using the estimated extinction to derive other quantities of the burst. Dust mixed with the stars can suggest quite large extinctions, but these are not applicable to the entire source. For the purpose of dereddening the $H-K$ color it is not crucial which model we employ, and we choose the screen here for simplicity. In Figs. 4(c) and 4(d) we show $\mathrm{CO}_{\mathrm{sp}}$ versus dereddened $H-K$ for the northern and southern slit points. Similarly, increasing the dust temperature reduces the estimated line-of-sight extinction for a given measured $J-H, H-K$ color. For the $H-K$ colors measured here (see below) the difference between 600 and $1000 \mathrm{~K}$ dust amounts to extinction differences of $A_{V} \sim 2 \mathrm{mag}$. As can be seen in Figs. 4(a) and 4(b), these are not large enough to change our conclusions, and indeed increasing the dust temperature requires a larger warm dust contribution and a correspondingly smaller contribution from young stars to the $K$-band continuum. In reflection nebulae, where single photon heating of small dust grains dominates, the $1-2 \mu \mathrm{m}$ continua can be fit with thermal dust models of $T \sim 1000 \mathrm{~K}$ (Sellgren 1983). A strong contribution from small grains then is equivalent, for our purposes, to increasing the temperature of the grains assumed to be in thermal equilibrium. Small grains are not expected to be important, however, since the line-tocontinuum ratios found for reflection nebulae by Sellgren and the strength of the $3.27 \mu \mathrm{m}$ emission feature in Arp 220 measured by Rieke et al. (1985) together imply that the contribution to the continuum from these grains is only about $6 \%$ at $2.2 \mu \mathrm{m}$. We have not explicitly removed this contribution from the measured continuum flux density since we are simply attempting to assess the maximum contribution of stars to the $K$-band light, and therefore it does not significantly effect our conclusions. A much larger contribution from small grains, in part replacing the emission assumed here to be from warm dust in thermal equilibrium, would only decrease the amount of $K$-band light produced by young stars and thus lower the importance of a starburst for the bolometric luminosity (see below).

Along with the data points, there are two simple models depicted in Figs. 4(c) and 4(d). The vertical dotted line in each figure represents the mixing curve of an old stellar population (the E/SO point) and a pure, red supergiant (the M4 I point). The dotted curves labeled "A," "B," and "C" represent the effects of adding warm $(600 \mathrm{~K})$ dust emission to these stellar population mixes.

Since there are strong indications that the power source may be highly concentrated in Arp 220 and thus the circumnuclear emission may have a different power source or population mix, we discuss the nuclear and off-nuclear points separately below.

\subsection{The Nuclear Spectra}

Mid-infrared observations by Becklin \& Wynn-Williams (1986) imply that the source size at $20 \mu \mathrm{m}$ is less than $1.5^{\prime \prime}$. Similarly, $1.49 \mathrm{GHz}$ maps by Condon et al. (1990) show that over $90 \%$ of the total radio emission mapped on $18^{\prime \prime}$ scales arises in the central 1.5", while high-resolution $15 \mathrm{GHz}$ maps (Sopp \& Alexander 1991) have 32\% of the emission arising on scales less than $0.14^{\prime \prime}$ in extent. The $15 \mathrm{GHz}$ radio peaks are also well aligned with the two infrared nuclei discovered by G90. Together, these results argue for a very compact source of luminosity in Arp 220 coincident with the infrared nuclei. The near-infrared spectra presented here likewise suggest that the peak in the strong $\mathrm{CO}$ absorption is centered on the nuclei. The $\mathrm{CO}$ indices measured on both the eastern and western nucleus of Arp 220 are larger than expected from a purely reddened old stellar population. There must therefore, be some other source of deep CO atmospheric absorption that contributes to the integrated $K$-band light. Red supergiant stars are a natural choice for this added spectral component. Figure 4(c) suggests that an equal mix of old stars plus M4 supergiant light, coupled with a 10\%-20\% contribution to the integrated $K$-band light from warm $(600$ $\mathrm{K})$ dust could match the strength of the $\mathrm{CO}$ index and the $H-K$ colors of both Arp 220 nuclei. If a typical late M-type supergiant has an absolute $K$-band magnitude of -11.9 mag (Elias et al. 1981) and if about 50\% of the $K$-band light is contributed by supergiants of this type, then the equivalent of approximately $5000 \mathrm{M} 4 \mathrm{I}$ stars are required in each of the Arp 220 nuclei.

Although the effects of free-free emission on the $K$-band continuum have not been included in Fig. 4, calculations of the free-free emission derived using the extinction-corrected $B \gamma$ fluxes as measured by Larkin et al. (1995) along with the theoretical $B \gamma$-to-free-free continuum ratio from Joy \& Lester (1988), imply the free-free contribution is less than $1 \%$ of the $K$-band flux of the nuclei.

At the positions of the Arp 220 nuclei, the $B \gamma$ equivalent 

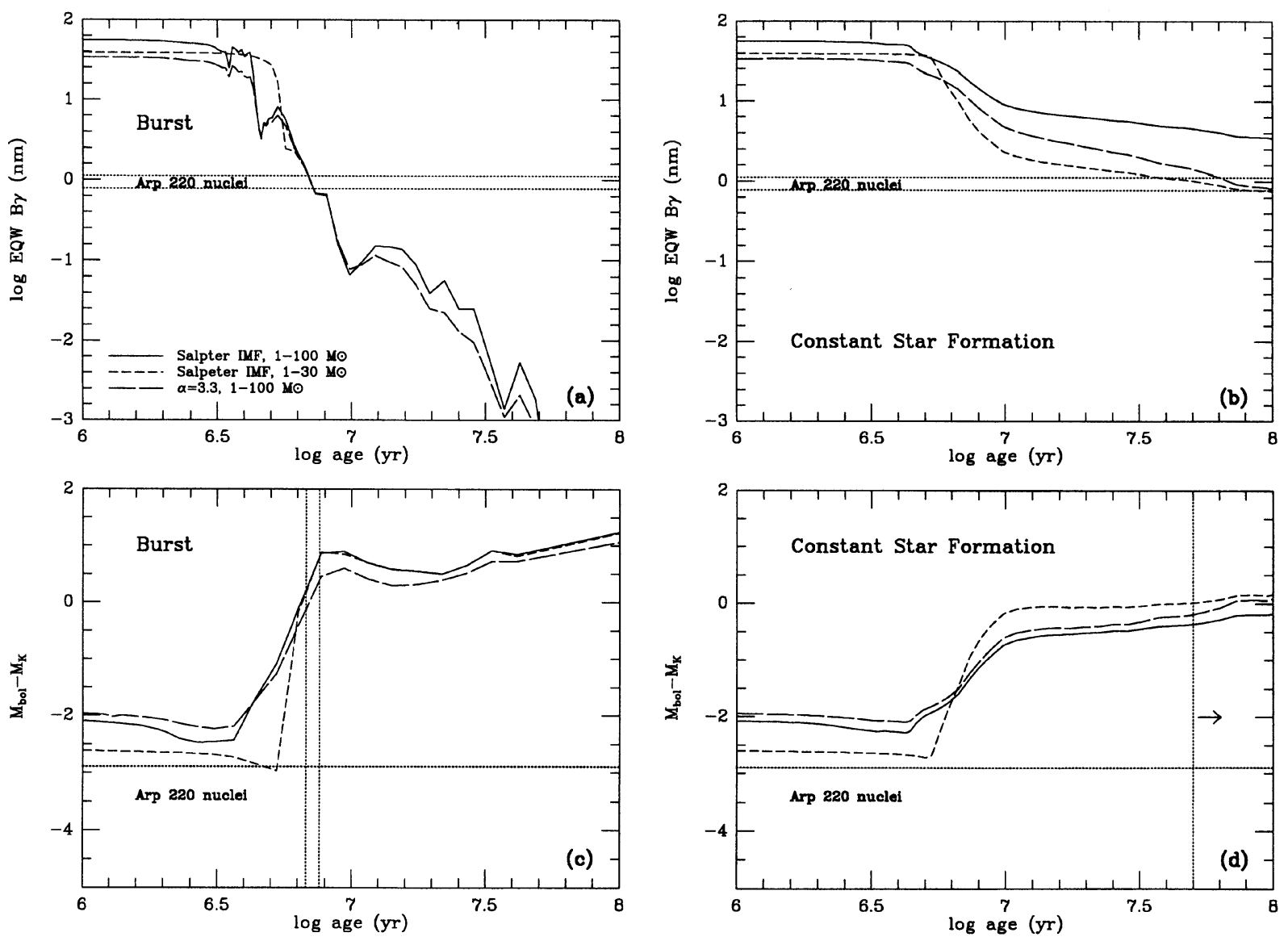

FIG. 5. Model curves tracing the evolution in the $B \gamma$ equivalent width and the quantity $M_{\text {bol }}-M_{K}$ with time for both a decaying starburst [(a) and (c)] and constant star formation (b) and (d) from the models of Leitherer \& Heckman (1995). The solid curve represents star formation following a Salpeter IMF over the $1-100 \mathscr{H}_{\odot}$ mass range. The short-dashed curve represents star formation following an IMF with the same slope, but truncated at $30 \mathscr{A b}_{\odot}$. The long-dashed curve represents star formation following an IMF with a slope of $\alpha=3.3$ over the $1-100 . \mathscr{H}_{\odot}$ mass range. In (a) and (b) the horizontal dotted lines represent the $B \gamma$ equivalent widths of the two near-infrared nuclei in Arp 220. The implied ages are 6.7-7.5 Myr for the burst models and greater than 32 Myr for the constant star formation models. In (c) and (d) these ages are indicated by vertical dotted lines. Horizontal dotted lines represent the approximate value of $M_{\text {bol }}-M_{K}$ for the nuclei, if each nucleus is responsible for $50 \%$ of the total energy output of Arp $220\left(7.5 \times 10^{11} L\right)$. Over the age ranges indicated by the $B \gamma$ equivalent widths, the starburst models with either a decaying burst or constant star formation fail by more than an order of magnitude to provide the bolometric energy of Arp 220.

widths are small, being only $1.0 \pm 0.1$ and $0.7 \pm 0.1 \mathrm{~nm}$ for the eastern and western nucleus, respectively. Correcting for a $10 \%$ contribution from dust emission, the intrinsic $B \gamma$ equivalent width is $\sim 1.1$ and $0.8 \mathrm{~nm}$ on the eastern nucleus and western nucleus, respectively, with respect to the stellar continuum. As we will see below, the ionizing flux from the starburst (as measured via the $B \gamma$ equivalent width) is well below that expected from a population of young stars responsible for a significant fraction of the bolometric luminosity in Arp 220.

In Figs. 5(a) and 5(b), the evolution in the $B \gamma$ equivalent width as a function of time for decaying and constant star formation models is shown. These figures have been reproduced from Figs. 49 and 50 of Leitherer \& Heckman (1995). Figure 5(a) represents the decay of an instantaneous starburst, and Fig. 5(b) represents star formation proceeding at a constant rate of $1 \mathscr{C l}_{\odot} / \mathrm{yr}$. In both plots, the solid line represents an initial mass function with a power-law slope of $\alpha=2.35$ integrated over the $1-100 \mathscr{C}_{\odot}$ range. The short- dashed line represents an initial mass function with the same slope, but with an upper mass cutoff of only $30 \mathscr{M}_{\odot}$. The long-dashed line corresponds to a solar neighborhood $(\alpha$ =3.3) IMF (Miller \& Scalo 1979) over the 1-100 $\mathscr{C b}_{\odot}$ range. Figure 5(a) suggests that the low $B \gamma$ equivalent widths seen in the Arp 220 nuclei can be reproduced by a decaying instantaneous starburst if the time since the burst has been about 7 Myr. This is independent of the IMF slope or initial mass cutoff. It is also comparable to the merger timescale for the two infrared nuclei calculated by G90. The constant star formation models of Fig. 5(b) require that the time since the onset of star formation be at least $32 \mathrm{Myr}$.

With these estimates of the allowed ages, it is possible to calculate the bolometric luminosity of the starburst(s). In Figs. 5(c) and 5(d) the evolution in time of the quantity $M_{\text {bol }}-M_{K}$ is shown. These figures are assembled from Figs. $7-8,11-12$, and 25-26 of Leitherer \& Heckman (1995). In Figs. 5(c) and 5(d), the starburst ages determined from Figs. 5(a) and 5(b) are indicated by vertical dotted lines. In these 
models, $M_{\text {bol }}=-2.5 \log \left(L_{\text {bol }} / L_{\odot}\right)+4.75$, and $L_{\text {bol }}$ is integrated from $50 \AA$ to $9 \mu \mathrm{m}$ (note that $L_{\text {bol }}$ is the emitted luminosity before reprocessing via thermal dust emission). Both the Arp 220 nuclei have an absolute $K$-band magnitude of approximately $-22.1 \mathrm{mag}$, after correcting for the extinctions implied by the Larkin et al. (1995) spectra. If approximately $90 \%$ of the light in each nucleus is stellar $\left(M_{K} \sim-22.0 \mathrm{mag}\right)$, and if each nucleus is responsible for $50 \%$ of the bolometric luminosity of Arp 220 $\left(L_{\text {bol }}=7.5 \times 10^{11} L_{\odot}\right)$ then each nucleus should have $M_{\text {bol }}-M_{K} \sim-2.9 \mathrm{mag}$. This is the horizontal dotted line in Figs. 5(c) and 5(d). For the decaying burst models over the period $\sim 7 \mathrm{Myr}$ after the onset of the burst, $0<M_{\text {bol }}-M_{K}<1$ mag, or about 3 mag larger than required if either nucleus is to generate $50 \%$ of the galaxy's energy output. Thus a decaying starburst in either nucleus can produce at most about $0.5 \times 10^{11} L_{\odot}$, or $3 \%$ of the total energy radiated by Arp 220 . For the constant star formation models the results are similar. Only for the truncated IMF starburst (upper mass limit equal to $30 \mathscr{L}_{\odot}$ ) does the $M_{\text {bol }}-M_{K}$ approach that of the Arp 220 nuclei. However, this only occurs at an age of about $5 \mathrm{Myr}$. At this time, the $B \gamma$ equivalent width should be approximately $40 \mathrm{~nm}$, a factor of 36 greater than what is measured on the eastern nucleus. If intermediate A-type stars are abundant, the $B \gamma$ emission line equivalent widths may have been underestimated by at most a factor of 2 , since these stars can have $B \gamma$ absorption of $<1 \mathrm{~nm}$. The large observed $\mathrm{CO}_{\mathrm{sp}}$, however, places an effective lower limit on the age of the burst (if the stars are coeval), since the red supergiant progenitors (15-30 $\mathscr{M}_{\odot}$ OB stars) must have time to evolve off the main sequence (Humphreys 1990). The decaying starburst model with the truncated IMF produces a $\mathrm{CO}_{\mathrm{sp}} \leqslant 0.1$ mag at an age of $5 \mathrm{Myr}$. The peak in the CO index does not occur until about $7 \mathrm{Myr}$ after the onset of the burst [C. Leitherer (private communication)].

Note, that if we mix the starburst [labelled SB in Fig. 4(c)] with the old stellar population, instead of a pure M4 I star as done above, the required warm dust contribution is reduced from $10 \%$ to essentially zero in the nuclei. The young stars themselves, however, only contribute approximately $50 \%$ of the near-infrared light in the nuclei making the bolometric contribution of the starburst correspondingly less (recall that above we assume that all the stellar light is associated with the burst). The discrepancies would then be even larger between the measured and predicted $M_{\text {bol }}-M_{K}$.

The starburst responsible for the strong $\mathrm{CO}$ absorption in the nuclei thus does not contribute a significant fraction of the bolometric luminosity in Arp 220. The measured $B \gamma$ equivalent widths are far too small to be from a starburst large enough to power the far-infrared emission through dust reradiation, even after correcting for dust emission and A-type star dilution. This is essentially the "ionizing photon deficit" in Arp 220 (e.g., Scoville et al. 1991). However, it may be possible that we have severely underestimated the extinction in the near infrared. For a starburst in each nucleus to produce $50 \%$ of the total infrared luminosity would require additional extinctions of about $4 \mathrm{mag}$ in the $K$ band, or visual extinctions of about $40 \mathrm{mag}$. These are much larger than those measured by Larkin et al. (1995), or those implied by the $H-K$ colors (see Fig. 4), but less than those estimated from the $9.7 \mu \mathrm{m}$ silicate absorption optical depth (Smith et al. 1989). We have direct evidence for young stars in the $K$-band spectra, but the extinction-corrected $K$-band light (where the extinctions have been estimated from nearinfrared emission-line flux ratios) is much smaller than that expected if the starburst produces the majority of the bolometric luminosity. The primary energy source apparently remains obscured even at $2.2 \mu \mathrm{m}$. This has most recently been suggested for the ultraluminous infrared galaxies as a class by Goldader $e t$ al. (1995). This obscured energy source may be an active nucleus or it may be a powerful yet very compact and hidden starburst (e.g., Condon et al. 1990).

\subsection{The Off-Nuclear Spectra}

At distances greater than $1.0^{\prime \prime}$ from either the eastern or western nucleus, the $\mathrm{CO}$ indices are consistent with those from old stellar populations. However, Figs. 4(c) and 4(d) suggest that there are additional points along both slits whose red $H-K$ colors and $\mathrm{CO}$ indices could be interpreted as the combination of warm dust emission coupled with measurable supergiant light in the $K$ band. In Fig. 4(c), the points corresponding to the positions about $0.5^{\prime \prime}$ and $0.9^{\prime \prime}$ east of the eastern nucleus, and in Fig. 4(d) the points corresponding to the positions directly below the eastern nucleus, as well as those about $0.7^{\prime \prime}$ and $1.5^{\prime \prime}$ east of this position, all have $H-K$ $>0.5 \mathrm{mag}$ (after dereddening) and $\mathrm{CO}_{\mathrm{sp}}>0.18 \mathrm{mag}$. This $\mathrm{CO}_{\mathrm{sp}}$ is well within the range seen in the nuclei of E/S0 galaxies, but the red $H-K$ colors of these spectra suggest a possible strong warm dust contribution in the $K$ band, and thus the intrinsic $\mathrm{CO}$ strength at these positions along the slit may be larger than the measured values.

Strong off-nuclear intrinsic CO absorption coupled with red $H-K$ colors imply a large population of young stars. Here, as in the nuclei, dust continuum emission can cause a significant underestimate of the intrinsic $B \gamma$ line strengths. Also if the line-of-sight extinction to these points has been underestimated, the intrinsic $H-K$ colors would be bluer, the warm dust contribution would be negligible, and the intrinsic $\mathrm{CO}_{\mathrm{sp}}$ would be consistent with old stars. For the southern slit points this would imply additional extinctions of about 2-5 visual magnitudes while for the northern slit points, additional extinctions of 4-7 visual magnitudes would be required.

The presence of strong $\mathrm{H} \beta$ absorption in the visual spectrum of Arp 220 (Rieke et al. 1985; Armus et al. 1989) indicates a large population of intermediate age $\left(\sim 10^{8} \mathrm{yr}\right)$ stars. Although the visual data are of low spatial resolution, the large extinctions to the nuclei suggest that these visual absorption features may be mostly circumnuclear in origin. The strong $\mathrm{H} \beta$ absorption would then be produced by stars which formed earlier than the supergiants detected on the nuclei themselves through their strong $K$-band emission. The shallow spectral indices $(\beta)$ seen off the nuclei and the $J-H$ and $H-K$ colors measured by Mazzarella et al. (1992) at radii of $2.0^{\prime \prime}-2.5^{\prime \prime}$ suggest warm dust emission on levels of about $15 \%$ of the $K$-band flux. This amount of dust emission is consistent with the locations of these points in Figs. 4(c) 
and 4(d), which have $\mathrm{CO}$ indices which are on average, below the E/SO locus. The dust at these distances from the nuclei may be heated by both the young stars in the nuclei (via single photon heating of small grains) as well as the older stars surrounding the nuclei which are responsible for the observed balmer absorption. Note that intermediate age stars will also will also decrease the measured $B \gamma$ emission equivalent width as discussed above.

If we model the Arp 220 system as a combination of a young $\left(\sim 10^{7}\right.$ yr old $)$ nuclear population and an older $\left(\sim 10^{8}\right.$ yr old) circumnuclear population, we can estimate the maximum contribution of star formation to the galactic bolometric luminosity. Although the model is not unique, it does explain both the strong nuclear $\mathrm{CO}$ absorption, the strong off-nuclear balmer absorption, and the red $H-K$ colors. Within a $5.0^{\prime \prime}$ diameter beam, Carico et al. (1990) measure $22 \mathrm{mJy}$ at $2.2 \mu \mathrm{m}$. If $6 \mathrm{mJy}(25 \%)$ of this comes from the nuclei themselves (G90), then $16 \mathrm{mJy}$ comes from the surrounding region. The extinction to the extended continuum source is likely (on average) to be much less than to the nuclei, and we adopt an $A_{V}=3 \mathrm{mag}$. If $80 \%$ of this is starlight, the absolute magnitude of the stars is $M_{K} \sim-22.9$ mag. At an age of $10^{8} \mathrm{yr}$, the decaying burst models of Leither \& Heckman (1995) have $1.0<M_{\mathrm{bol}}-M_{K}<1.5 \mathrm{mag}$. Thus, the stars surrounding the nuclei have $-21.9>M_{\text {bol }}>-21.4 \mathrm{mag}$, or $2.9 \times 10^{10}<L_{\text {bol }}<4.6 \times 10^{10}\left(L_{\odot}\right)$. This is only approximately $3 \%$ of the total energy output of Arp 220 .

Since the nuclei together can contribute up to $\sim 6 \%$ of the galactic bolometric luminosity, star formation within the central $5^{\prime \prime}$ is able to account for less than $10 \%$ of the infrared luminosity of Arp 220. This rather small contribution seems contradictory to previous estimates made by other authors, e.g., Rieke et al. (1985), who generate up to $50 \%$ of the bolometric luminosity in Arp 220 with flat, truncated IMF starburst models at ages of 30-100 Myr. However, Rieke et al. infer a large extinction $\left(A_{V}=10 \mathrm{mag}\right)$ over a large beam ( 8.7 arsec diameter) to constrain the model parameters. They calculate a starburst absolute magnitude of $M_{K}=-24.5$ mag. Since the $K$-band light is not highly concentrated in Arp 220, while the extinction is apparently peaked strongly on the nuclei, a large beam measurement assuming a large overall extinction will overestimate the potential contribution of a starburst. The simple, composite nuclear (young) plus circumnuclear (old) starburst model employed here uses extinctions to the near-infrared nuclei made from measurements at similar wavelengths (the $J-H$ and $H-K$ colors and the $P \beta$-to- $B \gamma$ emission-line flux ratios) and thus are not as dependent upon extrapolations over large wavelength intervals. Our simple model does imply, however, that the young stars which contribute to the near-infrared spectra are not the dominant power source in Arp 220.

\section{SUMMARY}

A near-infrared long-slit spectroscopic study of Arp 220 in the $K$-band atmospheric window has revealed spatially variable $\mathrm{CO}$ absorption and $B \gamma$ emission over the central seven arcseconds. In particular we find that:

(1) The two near-infrared nuclei have very strong stellar atmospheric $\mathrm{CO}$ absorption coupled with very red $H-K$ colors, implying significant contributions $(\sim 50 \%)$ to the $K$-band continua from young, red supergiant stars. These supergiant stars provide direct evidence for a starburst occurring approximately $10^{7} \mathrm{yr}$ ago in the Arp 220 nuclei.

(2) In contrast to the strong nuclear $\mathrm{CO}$ absorption, the equivalent width of the $B \gamma$ emission line is weak, with a relative maximum at the position of the eastern infrared nucleus of $1 \mathrm{~nm}$. There is no detectable $B \gamma$ emission in the southern slit data.

(3) Using the models of Leitherer \& Heckman (1995), we have estimated the contribution of a young $\left(t \sim 10^{7} \mathrm{yr}\right)$ nuclear starburst coupled with an older $\left(t \sim 10^{8}\right.$ yr $)$ circumnuclear population to be at most $10 \%$ of the total bolometric luminosity of Arp 220. Although the strong nuclear CO absorption and relatively weak $B \gamma$ emission provides direct evidence for a decaying starburst, the dominant energy source apparently remains hidden even at $2.2 \mu \mathrm{m}$.

The W. M. Keck Observatory is operated as a scientific partnership between the California Institute of Technology and the University of California. We thank the entire Keck Observatory staff, especially Wendy Harrison, for making these observations possible. In addition, we thank Tim Heckman and Claus Leitherer for many helpful discussions, and for making their model results available to us prior to publication. We also thank an anonymous referee for many helpful comments which strengthened the presentation of this paper. Infrared astronomy at Caltech is supported by grants from NASA and the NSF. This research has made use of the NASA/IPAC Extragalactic Database which is operated by the Jet Propulsion Laboratory, Caltech, under contract with NASA.

\section{REFERENCES}

Aaronson, M. A. 1977, Ph.D. thesis, Harvard University

Armus, L., Heckman, T. M., \& Miley, G. K. 1989, ApJ, 347, 727

Becklin, E. E., \& Wynn-Williams, C. G. 1986, in Star Formation in Galaxies, edited by C. J. Lonsdale (NASA Conference Publication 2466), p. 643 Black, J. H., \& van Dishoeck, E. F. 1987, ApJ, 322, 412

Carico, D. P., Sanders, D. B., Soifer, B. T., Mattews, K., and Neugebauer, G. 1990, AJ, 100, 70

Condon, J. J., Helou, G., Sanders, D. B., \& Soifer, B. T. 1990, ApJS, 73, 359

Doyon, R., Joseph, R. D., \& Wright, G. S. 1994, ApJ, 421, 101
Elias, J. H., Frogel, J. A., Humphreys, R. M., \& Persson, S. E. 1981, ApJ, 249, L55

Frogel, J. A., Persson, S. E., Aaronson, M., \& Matthews, K. 1978, ApJ, 220, 75

Goldader, J. D., Joseph, R. D., Doyon, R., \& Sanders, D. B. 1995, ApJ, 444, 97

Graham, J. R., Carico, D. P., Matthews, K., Neugebauer, G., Soifer, B. T., \& Wilson, T. D. 1990, ApJ, 354, L5

Humphreys, R. M. 1990, in Massive Stars in Starbursts, edited by C. Leitherer et al. (Cambridge University Press, New York), p. 45 
Joy, M., \& Lester, D. F. 1988, ApJ, 331, 145

Larkin, J. E., Graham, J. R., Matthews, K., Soifer, B. T., Beckwith, S., Herbst, T. M., \& Quillen, A. C. 1994, ApJ, 420, 159

Larkin, J. E., Armus, L., Knop, R. A., Matthews, \& Soifer, B. T. 1995, ApJ (in press)

Leitherer, K., \& Heckman, T. M. 1995, ApJS, 96, 9

Lonsdale, C. J., Smith, H. E., \& Lonsdale, C. J. 1993, ApJ, 405, L9

Lonsdale, C. J., Diamond, P. J., Smith, H. E., \& Lonsdale, C. J. 1995, submitted to ApJ

Matteucci, F., \& Padovani, P. 1993, ApJ, 419, 485

Matthews, K., \& Soifer, B. T. 1994, in Infrared Astronomy with Arrays: The Next Generation, edited by I. McLean (Kluwer, Dordrecht), p. 239

Mazzarella, J. M., Soifer, B. T., Graham, J. R., Hafer, C. I., Neugebauer, G., \& Matthews, K. 1992, AJ, 103, 413

Miller, G. E., \& Scalo, J. M. 1979, ApJS, 41, 513
Ridgway, S. E., Wynn-Williams, C. G., \& Becklin, E. E. 1994, ApJ, 428, 609

Rieke, G. H., Cutri, R. M., Black, J. H., Kailey, W. F., McAlary, C. W., Lebofsky, M. J., \& Elston, R. 1985, ApJ, 290, 116

Salpeter, E. E. 1955, ApJ, 121, 161

Sanders, D. B., Soifer, B. T., Elias, J., Madore, B., Matthews, K., Neugebauer, G., \& Scoville, N. 1988 ApJ, 325, 74

Scoville, N. Z., Sargent, A. I., Sanders, D. B., \& Soifer, B. T. 1991, ApJ, 366, L5

Sellgren, K. W. 1983, Ph.D. thesis, California Institute of Technology

Shaya, E. J., Dowling, D. M., Currie, D. G., Faber, S. M., \& Groth, E. J. 1994, AJ, 107, 1675

Smith, C. H., Aitken, D. K., \& Roche, P. F. 1989, MNRAS, 241, 425

Soifer, B. T., Boehmer, L., Neugebauer, G., \& Sanders, D. B. 1989, AJ, 98, 766

Sopp, H. M., \& Alexander P. 1991, MNRAS, 251, 112 